\title{
Syndromic versus Laboratory Diagnosis of Sexually Transmitted Infections in Men in Moshi District of Tanzania
}

\author{
Yuwei Cheng, ${ }^{1}$ Elijah Paintsil $\left(\mathbb{D},{ }^{2}\right.$ and Musie Ghebremichael ${ }^{3}{ }^{3}$ \\ ${ }^{1}$ Department of Mathematical and Computer Sciences, College of the Holy Cross, Worcester, MA, USA \\ ${ }^{2}$ Department of Pediatrics, Yale University School of Medicine, New Haven, CT, USA \\ ${ }^{3}$ Harvard Medical School, Ragon Institute of MGH, MIT and Harvard, Cambridge, MA, USA \\ Correspondence should be addressed to Musie Ghebremichael; musie_ghebremichael@dfci.harvard.edu
}

Received 31 October 2019; Accepted 26 December 2019; Published 7 February 2020

Academic Editor: Seble Kassaye

Copyright (C) 2020 Yuwei Cheng et al. This is an open access article distributed under the Creative Commons Attribution License, which permits unrestricted use, distribution, and reproduction in any medium, provided the original work is properly cited.

\begin{abstract}
The syndromic diagnosis of sexually transmitted infections (STIs) is widely recognized as the most practical, feasible, and costeffective diagnostic tool in resource-limited settings. This study assessed the diagnostic accuracy of syndromic versus laboratory testing of STIs among 794 men randomly selected from the Moshi district of Tanzania. Participants were interviewed with a questionnaire that included questions on history of STIs symptoms. Blood and urine samples were taken from the participants for laboratory testing. Only $7.9 \%$ of the men reported any symptoms of STI; however, $46 \%$ of them tested positive for at least one STI. There was little agreement between syndromic and laboratory-confirmed diagnoses, with low sensitivity $(0.4 \%-7.4 \%)$ and high specificity (96\%-100\%) observed for each individual symptom. The area under the receiver-operating curve was 0.528 (95\% CI: $0.505-0.550$ ), indicating that the syndromic approach has a $52.8 \%$ probability of correctly identifying STIs in study participants. In conclusion, whenever possible, laboratory diagnosis of STI should be favored over syndromic diagnosis.
\end{abstract}

\section{Introduction}

AIDS continues to be one of the leading causes of death in sub-Saharan Africa [1]. Among the 36.9 million people living with HIV globally, 53\% lived in sub-Saharan Africa [2]. Sexually transmitted infections (STIs) facilitate the transmission, disease progression, and treatment outcomes of HIV [3-5]. Moreover, people living with HIV (PLWH) have an increased prevalence of other STIs [6]. In subSaharan Africa, high incidence of untreated STIs has been associated with an increased rate of HIV transmission [7]. The World Health Organization (WHO) reported that other STIs such as syphilis and HSV-2 increase a person's risk of acquiring HIV infection by more than three-fold [8]. Thus, timely recognition, management, and prevention of STIs are critical for prevention of HIV acquisition.

Although superior in terms of reliability, laboratory diagnosis of STIs is time-consuming, cost-prohibitive, and requires technology and capacity, which makes its routine use difficult in resource-limited countries. Most of these countries have a high burden of STIs; however, they lack the technical expertise, specialist physicians, and laboratory setup for the diagnosis of these STIs [9]. Furthermore, in situations where laboratory capacity exist, testing may be outsourced to regional facilities and obtaining test results may take up to several weeks. By contrast, syndromic case management algorithms provide an immediate result, allowing for on-site counseling and point-of-care treatment. Furthermore, syndromic diagnosis is feasible and economical in resource-limited countries; it costs less than a fifth of the cost of laboratory-based testing [10]. In 2001, the WHO introduced an updated algorithm for syndromic case management that uses decision trees for the most common signs and symptoms of STIs [11]. Based on the patient's symptoms and gender, different decision-tree diagrams are used. However, these symptoms may be subjective, variable among patients, and a patient with an STI may not manifest overt symptoms. Thus, syndromic diagnosis may miss individuals with asymptomatic STIs [12]. 
Syndromic diagnosis of STIs is popular in most healthcare systems in sub-Saharan Africa. Nevertheless, ongoing discussions regarding its effectiveness have persisted for years [13]. Several studies have investigated the utility of the syndromic method, especially focusing on target populations such as young women and sex workers [13-15]. Most of these studies were conducted in STI clinics or among particular groups (such as female sex workers), thereby introducing potential biases from convenient sampling. Thus, the findings from these studies may not be generalized to the other populations and clinical settings. This emphasized the need for populationbased studies in sub-Saharan Africa to test for the validity of the syndromic approach versus laboratory-based testing for STIs.

The prevalence of transactional sex in sub-Saharan Africa is high, with men being the perpetuators. Adolescent girls and young women who engage in informal sexual exchange for housing, money, and education are at an increased risk of acquiring STIs from men [16]. Wamoyi et al. found that transactional sex is associated with acquisition of HIV; adolescent girls and young women who engage in transactional sex in sub-Saharan Africa are 50\% more likely to be infected with HIV, while the findings for men remain inconclusive [17]. Moreover, sub-Saharan African men have higher AIDS-related death, lower awareness, and treatment coverage of HIV compared with women from the region $[2,18]$. Despite these statistics, there are few studies from the region focusing on STIs in men. Thus, more studies are needed to elucidate the relationship between STI symptoms and tests among men from the region. Our study addresses this need by evaluating the diagnostic accuracy of the commonly used syndromic approach of STIs in men in the Moshi urban district of Tanzania.

\section{Methods}

2.1. Study Participants. This study is based on secondary analysis of data collected during the Moshi Infertility Survey. The rationale, organization, and recruitment for the study have been described in detail elsewhere $[12,19]$. In brief, the survey was conducted from November 2002 to March 2003 in the Moshi urban district of Tanzania and involved a twostage sampling. During the first stage of sampling, 150 clusters of households were selected. In the second stage of sampling, 18 households were randomly selected to participate in the survey from each of the 150 clusters. A total of 2,019 women who were residents of the selected households and 794 men who were listed as husbands or partners of the women were interviewed. Information was collected on sociodemographic characteristics, STIs symptoms, and high-risk sexual behaviors. Blood samples were drawn to test for HIV-1, herpes simplex virus type 2 (HSV-2), and syphilis. Urine samples were obtained to test for chlamydia, gonorrhea, mycoplasma, and trichomonas STIs. The study protocol was approved by the Ethics Committees of the Kilimanjaro Christian Medical Centre, the Tanzania National Institute for Medical Research, the Institutional Review Boards of the Harvard Chan School of Public Health, the University of Maryland, and the Centers for Disease Control and Prevention (CDC), Atlanta, Georgia.

\subsection{Study Variables}

2.2.1. Symptoms of STIs. STI symptoms included at least one of the following: abdominal pain, genital discharge, foul smell in the genital area, excessive genital secretions, swellings in the genital area, itching in the genital area, burning pain on micturition, pain during intercourse, and genital ulcers.

2.2.2. Laboratory Tests of STIs. Blood samples were collected for HIV-1, HSV-2, and syphilis testing. HIV-1 infection was determined by using two enzyme-linked immunosorbent assays (ELISAs). Indeterminate results were resolved by Western blot (Genetic Systems HIV-1 Western blot; BioRad Laboratories, Redmond, WA). Antibodies to HSV-2 were detected using type-specific HSV-2 enzyme immune assay (EIA) (HerpeSelect 2 ELISA, Focus Technologies, Cypress, CA) according to the manufacturer's instructions. Active or recent syphilis was diagnosed if the serum was reactive to both the rapid plasma reagin card test (MacroVue; Becton-Dickinson, Cockysville, MD) and the Treponema pallidum hemagglutination assay (TPHA) (Wellcosyph HA; Murex Biotech Ltd., U.K.). A positive TPHA test alone was interpreted as evidence of a past infection.

Urine samples were tested for Chlamydia trachomatis, Neisseria gonorrhoeae, Trichomonas vaginalis, and Mycoplasma genitalium by using a real-time multiplex polymerase chain reaction (M-PRC) assay with primers and probe sets previously published [19]. In brief, all urine specimens were pooled in groups of 3, and DNA was extracted using the Qiagen Viral RNA kit (Qiagen), which is recommended for extraction of DNA from urine specimens. DNA was then extracted from all specimens falling within positive pools and tested by the M-PCR assay. Sequence-specific detection of M-PCR amplification products was based on TaqMan technology and was performed using the Rotor-Gene 3,000 instrument (Corbett Research, Australia). M-PCR amplifications were performed in $50-\mu \mathrm{L}$ reaction tubes using $25 \mu \mathrm{L}$ of sample DNA. A final concentration of 1X PCR Gold buffer (Applied Biosystems), $4 \mathrm{mmol} / \mathrm{L} \mathrm{MgCl}_{2}$, and $200 \mu \mathrm{M}$ each of dATP, dGTP, dCTP, and dUTP were used. Two units of AmpliTaq Gold DNA polymerase (Applied Biosystems) and one unit of uracil-N-glycosylase (UNG; Applied Biosystems) were used per $50-\mu \mathrm{L}$ reaction. Amplification was performed using the following parameters: $95^{\circ} \mathrm{C}$ for 10 minutes and $50^{\circ} \mathrm{C}$ for 2 minutes, followed by 50 cycles at $95^{\circ} \mathrm{C}$ for 20 seconds and $60^{\circ} \mathrm{C}$ for 60 seconds. Positive controls were prepared from cultures using the Qiagen (Valencia, CA) mini-DNA kit for N. gonorrhoeae, C. trachomatis, and $T$. vaginalis. $M$. genitalium-positive DNA control was obtained from the ATCC (catalog no. 33530D). The real-time M-PCR assay allows for detection over a broad range of target concentrations with analytical sensitivities of one to 10 genomic copies for M. genitalium and N. gonorrhoeae, 0.01 to 0.1 genomic copies for $T$. vaginalis, and the 


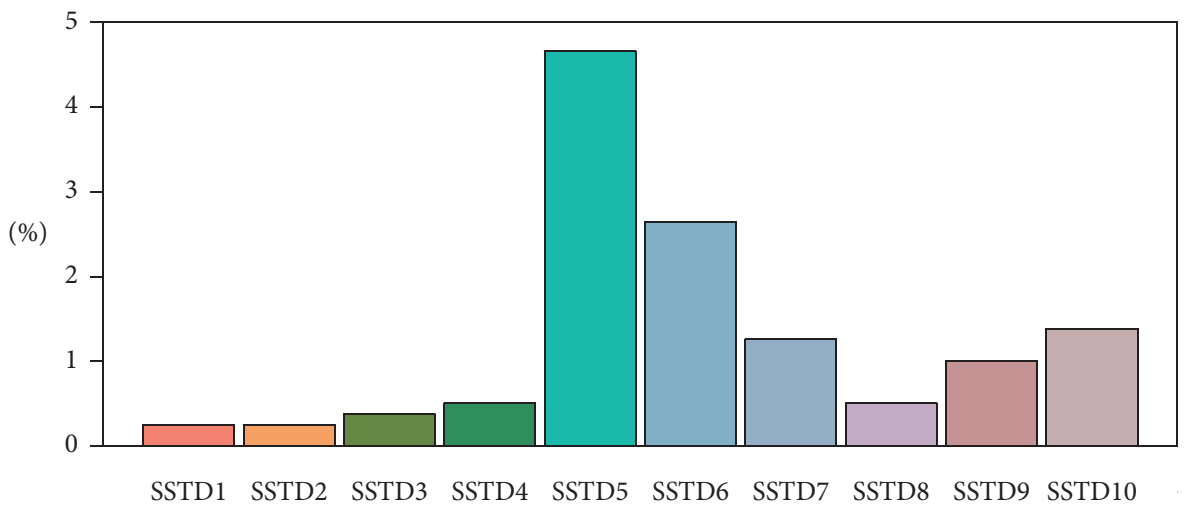

(a)

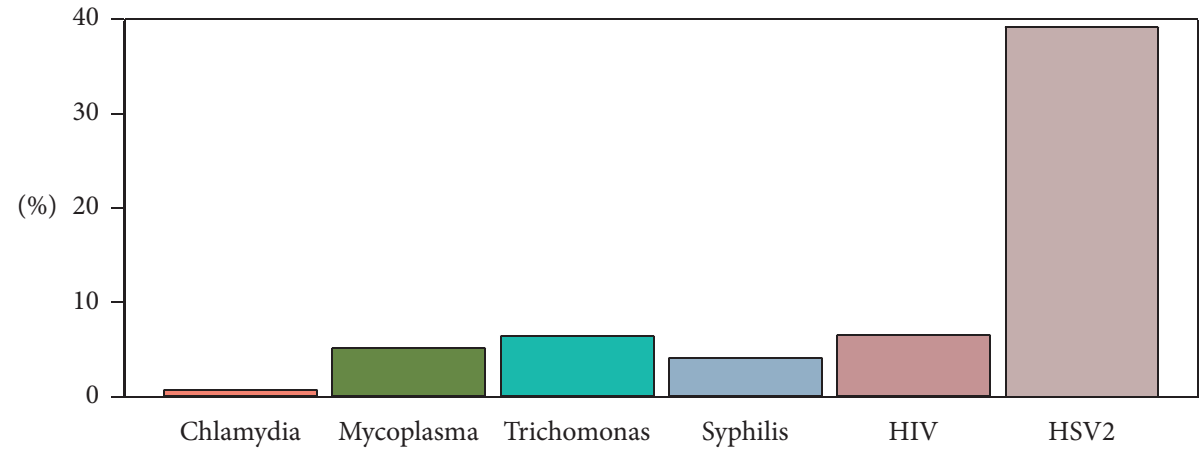

(b)

FIGURE 1: Prevalence of self-reported STIs symptoms and laboratory-confirmed STIs (SSTD1: lower abdominal pain, SSTD2: abnormal genital discharge, SSTD3: foul smell in the genital area, SSTD4: excessive genital secretions, SSTD5: swelling of lymph nodes in the genital area, SSTD6: itching in the genital area, SSTD7: burning pain on micturition, SSTD8: pain during intercourse, SSTD9: genital ulcers and open sores, and SSTD10: other unclassified symptoms).

equivalent of 0.01 to 0.1 inclusion-forming unit for $C$. trachomatis.

\subsection{Statistical Analysis. Statistical graphs and descriptive} measures (such as mean, median, standard deviation, interquartile range, frequencies, and percentages) were used to summarize the data. Sensitivity and specificity together with their corresponding 95\% confidence intervals were calculated to assess the predictive accuracy of each STIs symptom. Kendall's tau-b was used to measure agreements between patient-reported STIs symptoms and laboratoryconfirmed STIs. The area under the ROC curve (AUROC) was used to evaluate the overall diagnostic accuracy of the syndromic diagnosis of STIs. The analysis was conducted using the $\mathrm{R}$ programming language.

\section{Results}

This study assessed the effectiveness of syndromic versus laboratory testing of STIs among 794 men randomly selected from Moshi district of Tanzania. The average age of the men was 37 years, with a standard deviation of 8.9 years. Fortyfour percent $(44 \%)$ of the participants were in the 31 - to 40 year age-group. Only one-third of the participants had a secondary or higher education. Fifty-two percent (52\%) worked as either skilled or unskilled laborers, while $20 \%$ were unemployed. Most of the men (60\%) had their first sex before their $20^{\text {th }}$ birthday; only $16 \%$ of them had their first sexual intercourse at marriage or with a cohabiting partner. Most of the participants were in a monogamous relationship for the last 12 months (88\%) or 3 years (78\%). Moreover, $80 \%$ of the participants did not use condom during sexual intercourse in the previous 12 months.

Among the study participants, only $7.9 \%$ reported any symptoms, while $46 \%$ tested positive for at least one STI, suggesting that many of the men with STIs have asymptotic infections. Figure 1 shows the rates of STIs symptoms and tests among the men included in the analysis. The highest infection rate was for HSV-2 (39\%), followed by HIV-1 (7\%), trichomonas (6\%), and mycoplasma (5\%). The prevalence of other STIs such as syphilis and chlamydia was below $5 \%$. None of the participants tested positive for gonorrhea. The most prevalent STIs symptom was swelling in the genital area $(4.7 \%)$, followed by itching in the genital area $(2.7 \%)$, burning pain on micturition (1.3\%), genital ulcers or sores $(1.0 \%)$, excessive genital secretions $(0.5 \%)$, pain during sex $(0.5 \%)$, smell from the genital area $(0.4 \%)$, lower abdominal pain $(0.3 \%)$, abnormal genital discharge $(0.4 \%)$, and other nonspecific symptoms (1.4\%). The prevalence of STIs symptoms among men tested positive for each STI is presented in Figure 2. Multiple STI symptoms were reported 


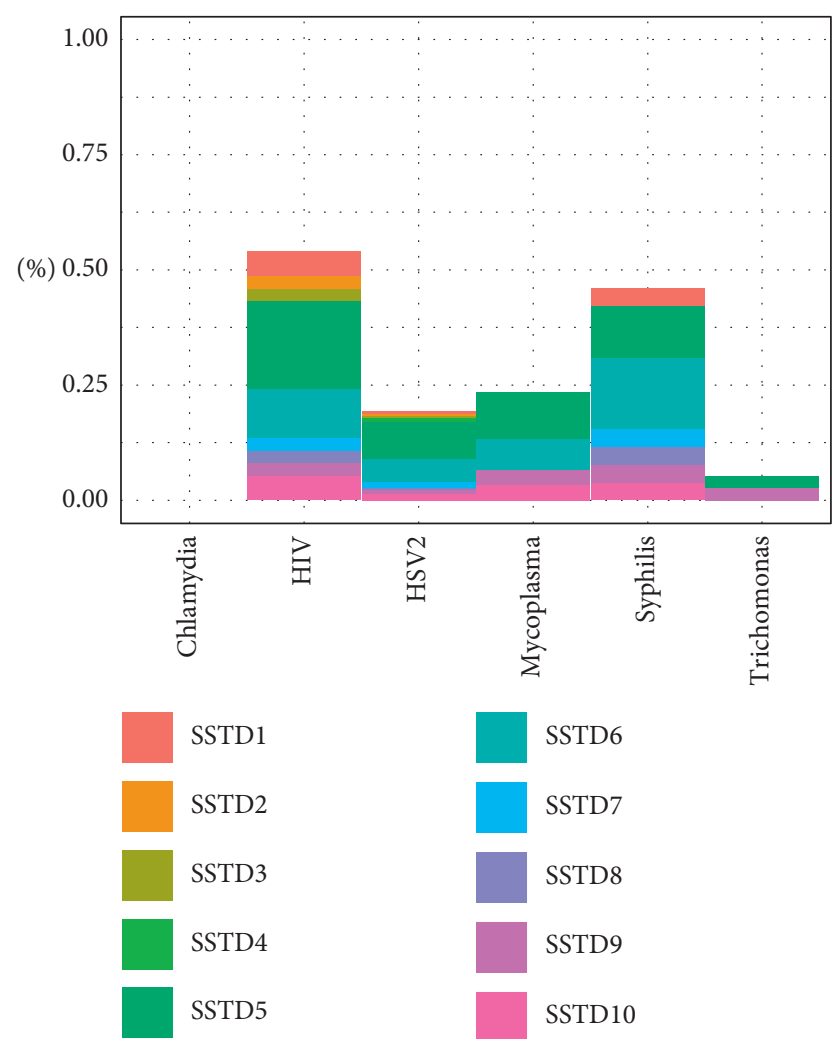

FIgURe 2: Prevalence of self-reported STIs symptoms among men tested positive for STIs (SSTD1: abdominal pain, SSTD2: abnormal genital discharge, SSTD3: foul smell in the genital area, SSTD4: excessive genital secretions, SSTD5: swelling of lymph nodes in the genital area, SSTD6: itching in the genital area, SSTD7: burning pain on micturition, SSTD8: pain during intercourse, SSTD9: genital ulcers, and SSTD10: other unclassified symptoms).

among men tested for HIV-1, HSV-2, syphilis, and mycoplasma. Men who tested positive for chlamydia were asymptomatic, and those who were positive for trichomonas on the average reported two symptoms. Swelling of lymph nodes in the genital area was a common symptom of STIs. Figure 3 displays a heat map of the correlation coefficients between laboratory-confirmed STI test results and patientreported STI symptoms. The coefficients were relatively low (range $=-0.05-0.24)$, suggesting no strong associations between STI symptoms and the laboratory-confirmed STI test results.

Figure 4 illustrates the sensitivity (true-positive rate) and specificity (true-negative rate) of each STI symptom and the combined symptoms. For each of the STI symptoms, sensitivity tended to be very low, with the maximum being $7.4 \%$ (swelling of lymph nodes in the genital area) and the minimum being $0.4 \%$ (abnormal genital discharge and smelling in the genital area). Specificities were all above 95\%, ranging from $96 \%$ to $100 \%$. The combined STI symptoms, with a positive response representing having at least one STI symptom, also had a low sensitivity 0.112 (95\% CI: $0.074-0.15)$ and a high specificity of 0.925 (95\% CI: $0.892-0.949$ ). These results suggest that the combined STI symptoms correctly identified 11 of 100 men with laboratory-confirmed STIs and resulted in an $89 \%$ false-negative

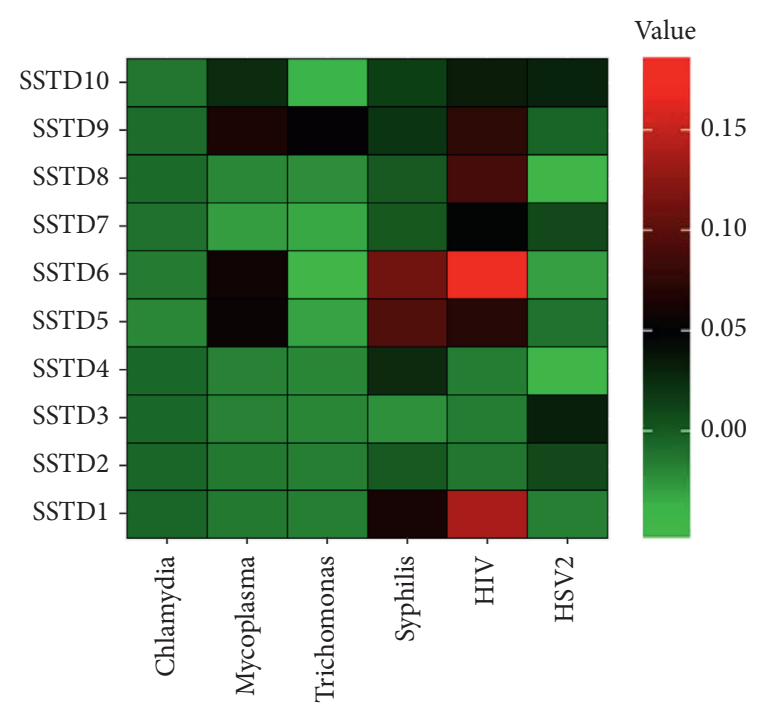

FIGURE 3: Heat map of Kendall's tau-correlation coefficients between STIs symptoms and laboratory-confirmed STIs (SSTD1: lower abdominal pain, SSTD2: abnormal genital discharge, SSTD3: foul smell in the genital area, SSTD4: excessive genital secretions, SSTD5: swelling of lymph nodes in the genital area, SSTD6: itching in the genital area, SSTD7: burning pain on micturition, SSTD8: pain during intercourse, SSTD9: genital ulcers and open sores, and SSTD10: other unclassified symptoms).

rate. Similarly, the combined STI symptoms correctly identified 92 of 100 healthy men and resulted in an $8 \%$ falsepositive rate. The reported numbers of STI symptoms were summed to obtain the total number of STI symptoms for each participant. The receiver-operating characteristic (ROC) curve was then constructed using sensitivity and specificity obtained from every possible symptom threshold. The area under the receiver-operating curve (AUROC) was 0.528 (95\% CI: 0.505-0.550), indicating that the syndromic approach would correctly identify an infected male patient from a healthy one only $52.8 \%$ of the time. Thus, the STI symptoms are no better than a random classifier which has an AUROC of 0.50 .

\section{Discussion}

This study investigated the diagnostic accuracy of syndromic diagnosis of STIs relative to laboratory-based diagnosis among men in Moshi district of Tanzania. The study cohort included men who were enrolled in a community-based survey. Forty-six percent of the men tested positive for at least one STI, while only $7.9 \%$ reported at least one STI symptom. The most prevalent STI among the study participants was HSV-2 (around 39\%), followed by HIV (7\%), trichomonas $(6 \%)$, and mycoplasma (5\%). The prevalence rates of other STIs were syphilis (4\%) and chlamydia (1\%). The high prevalence of HSV-2 may be partly due to the fact that the antibody test could not distinguish between acute and past infections. The prevalence of STI symptoms was below 5\%, with the most prevalent STI symptoms being swelling of the lymph nodes in the genital area (4.7\%) and itching in the genital area (2.7\%). We found little agreement 

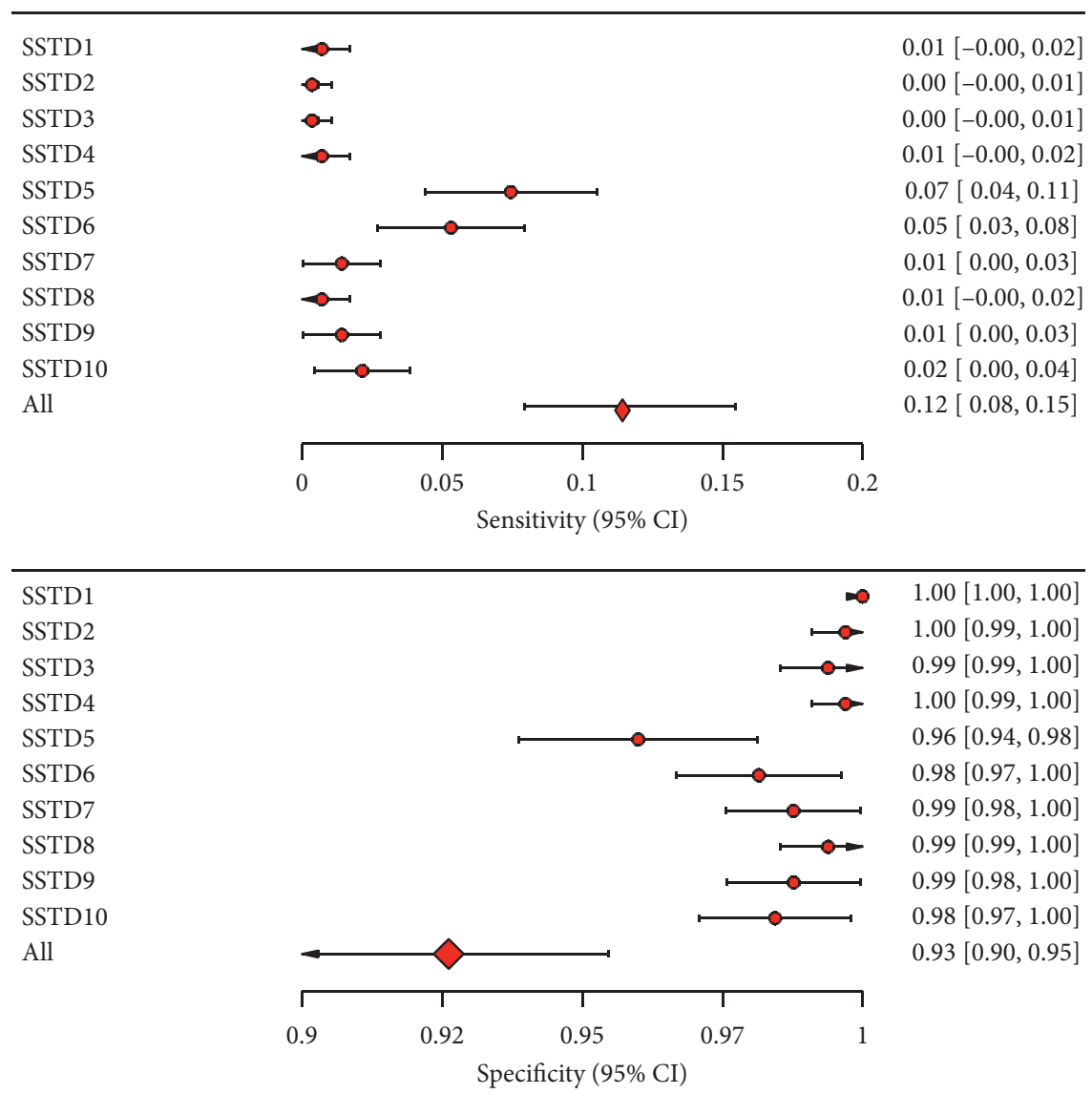

FIGURE 4: Sensitivities and specificities of STIs symptoms together with their 95\% exact confidence intervals (SSTD1: lower abdominal pain, SSTD2: abnormal genital discharge, SSTD3: foul smell in the genital area, SSTD4: excessive genital secretions, SSTD5: swelling of lymph nodes in the genital area, SSTD6: itching in the genital area, SSTD7: burning pain on micturition, SSTD8: pain during intercourse, SSTD9: genital ulcers and open sores, and SSTD10: other unclassified symptoms).

between patient-reported symptoms and laboratory test results for all the STIs considered in the study. Interestingly, itching in the genital area and swelling of lymph nodes in the genital area were associated with STIs compared with the other symptoms we considered.

To evaluate the performance of syndromic diagnosis of STIs, we calculated the sensitivity and specificity for each individual symptom and the combined STI symptoms. For each of the individual STI symptoms, sensitivity was low (ranged from $0.4 \%$ to $7.4 \%$ ), while specificity was relatively high (ranged from $96 \%$ to $100 \%$ ). In this context, the low sensitivity suggests that there is a low probability that a syndromic test will correctly detect an STI in a participant (i.e., there is a high false-negative rate). On the other hand, the high specificity observed in this population-based study suggests a high true-negative rate. The combined STI symptoms, which measure whether a participant has at least one STI symptom, also have very low sensitivity and high specificity. The area under the ROC curve was 0.528 , suggesting that the syndromic method can only correctly explain $52.8 \%$ of STIs in study participants. Almost half of the time, this approach gives an inappropriate diagnosis.

Studies published in the last decade have yielded conflicting results regarding the effectiveness of syndromic diagnosis of STIs. A study in Delhi by Choudhry et al. found the sensitivity of genital discharge syndrome (GDS) was high for Neisseria gonorrhoeae and Chlamydia trachomatis $(96 \%$ and $91 \%$, respectively), whereas the specificity for these STIs was relatively lower ( $76 \%$ and $72 \%$, respectively) [20]. They also found relatively low sensitivity and high specificity of genital ulcer syndrome for herpes simplex virus-2 (HSV-2). Another study by Grijsen et al. indicated that sensitivity tends to be low and specificity high for asymptomatic infection; $67 \%$ of urethritis cases in men and 59\% of cervicitis cases in women were missed using the syndromic approach [21]. Similar results were reported in a study by Theodora et al. with adequate accuracy for urethral discharge and genital ulcer disease syndromes, but the use of syndromic diagnosis was, however, limited by asymptomatic infections such as chlamydia and gonorrhea in females [22]. In our study, there were not gonorrhea cases; this may be due to the population sampling instead of enrolling patients from STI clinics.

Compared with previous studies, our study has advantages in terms of the sample size and comprehensiveness. Unlike many studies on the performance of syndromic versus laboratory-based diagnosis of STIs, we used population-based study and avoided the potential biases associated with sampling specific groups such as STI clinic attendees or sex workers. However, there are still several limitations of 
our study. The study included only men who gave consent. Incomplete cases could also lead to a biased result if there was a systematic difference between men who gave consent and those who did not. Furthermore, since symptoms are selfreported, some information from participants may remain underreported considering the sensitivity of STIs. Moreover, HSV-2 antibody positivity without concurrent symptoms ascribed to HSV has no clinical utility. The antibody testing might reflect past and resolved HSV-2 infections. Although we did not have HIV viral load testing, since HIV is a chronic nonrelapsing life-long infection, one can use antibody positivity as infectious state.

\section{Conclusion}

The use of syndromic diagnosis of STIs is pervasive in resource-limited countries because of the ease of implementation, accessibility, and low-cost. However, its performance is woefully inadequate, and there is a need to improve its sensitivity with other clinical correlates or gradually adopt laboratory testing for STI management in resource-limited settings.

\section{Data Availability}

The dataset used in the manuscript is available from the corresponding author on reasonable request.

\section{Conflicts of Interest}

The authors declare that they have no conflicts of interest.

\section{Authors' Contributions}

Y.C. analyzed data and wrote the manuscript. M.G. conceived and designed the study, wrote the manuscript, and provided guidance on data analyses and interpretation of the findings. E.P. reviewed the manuscript. All authors read and approved the final manuscript.

\section{Acknowledgments}

The study was supported by grants from Harvard University Center for AIDS Research (HU CFAR NIH/NAIDS P30-AI 060354) and the Ragon Institute of MGH, MIT and Harvard. The authors thank Dr. Shannon Stock for her helpful suggestions and comments.

\section{References}

[1] P. J. V. Vuuren, "Factsheet: Africa's leading causes of death. Africa check," 2019, http://africacheck.org/factsheets/factsheetafricas-leading-causes-death/.

[2] UNAIDS, UNAIDS DATA 2018, Joint United Nations Programme on HIV/AIDS (UNAIDS), Geneva, Switzerland, 2018.

[3] H. Ward and M. Rönn, "Contribution of sexually transmitted infections to the sexual transmission of HIV," Current Opinion in HIV and AIDS, vol. 5, no. 4, pp. 305-310, 2010.

[4] H. M. Chun, R. J. Carpenter, G. E. Macalino, and N. F. CrumCianflone, "The role of sexually transmitted infections in HIV-1 progression: a comprehensive review of the literature,"
Journal of Sexually Transmitted Diseases, vol. 2013, Article ID 176459, 15 pages, 2013.

[5] S. C. Kalichman, J. Pellowski, and C. Turner, "Prevalence of sexually transmitted co-infections in people living with /AIDS: systematic review with implications for using HIV treatments for prevention," Sexually Transmitted Infections, vol. 87, no. 3, pp. 183-190, 2011.

[6] S. R. Galvin and M. S. Cohen, "The role of sexually transmitted diseases in HIV transmission," Nature Reviews Microbiology, vol. 2, no. 1, pp. 33-42, 2004.

[7] L. F. Johnson, R. E. Dorrington, D. Bradshaw, and D. J. Coetzee, "The role of sexually transmitted infections in the evolution of a South African HIV epidemic," Tropical Medicine \& International Health, vol. 17, no. 2, pp. 161-168, 2012.

[8] Coetzee, Sextually Transmitted Infections (STIs), WHO, Geneva, Switzerland, 2012.

[9] W. K. Bosu, "Syndromic management of sexually transmitted diseases: is it rational or scientific?," Tropical Medicine \& International Health, vol. 4, no. 2, pp. 114-119, 1999.

[10] G. La Ruche, F. Lorougnon, and N. Digbeu, "Therapeutic algorithms for the management of sexually transmitted diseases at the peripheral level in Côte d'Ivoire: assessment of efficacy and cost," Bulletin of the World Health Organization, vol. 73, no. 3, pp. 305-313, 1995.

[11] World Health Organization, Guidelines for the Management of Sexually Transmitted Infections, WHO, Geneva, Switzerland, 2001.

[12] M. Ghebremichael, "The syndromic versus laboratory diagnosis of sexually transmitted infections in resource-limited settings," ISRN AIDS, vol. 2014, Article ID 103452, 5 pages, 2014.

[13] S. C. Francis, T. N. Mthiyane, K. Baisley et al., "Prevalence of sexually transmitted infections among young people in South Africa: a nested survey in a health and demographic surveillance site," PLoS Medicine, vol. 15, no. 2, Article ID e1002512, 2018.

[14] L. O. Mukenge-Tshibaka, M. Alary, C. M. Lowndes et al., "Syndromic versus laboratory-based diagnosis of cervical infections among female sex workers in Benin," Sexually Transmitted Diseases, vol. 29, no. 6, pp. 324-330, 2002.

[15] C. Mathews, A. van Rensburg, and N. Coetzee, "The sensitivity of a syndromic management approach in detecting sexually transmitted diseases in patients at a public health clinic in Cape Town," South African Medical Journal, vol. 88, no. 10, pp. 1337-1340, 1998.

[16] Centers for Disease Control and Prevention, "How STDs impact women differently from men," 2018, http://www.cdc. gov/nchhstp/newsroom/docs/factsheets/stds-women.pdf?s_ CID=tw_STD0170644.

[17] J. Wamoyi, K. Stobeanau, N. Bobrova, T. Abramsky, and C. Watts, "Transactional sex and risk for HIV infection in subSaharan Africa: a systematic review and meta-analysis," Journal of the International AIDS Society, vol. 19, no. 1, p. 20992, 2016.

[18] L. G. Johnston, M. L. Sabin, D. Prybylski et al., "The importance of assessing self-reported HIV status in biobehavioural surveys," Bulletin of the World Health Organization, vol. 94, no. 8, pp. 605-612, 2016.

[19] M. Ghebremichael and E. Paintsil, "High risk behaviors and sexually transmitted infections among men in Tanzania," AIDS and Behavior, vol. 15, no. 5, pp. 1026-1032, 2011.

[20] S. Choudhry, V. Ramachandran, S. Das, S. Bhattacharya, and N. Mogha, "Pattern of sexually transmitted infections and performance of syndromic management against etiological diagnosis in patients attending the sexually transmitted infection clinic of a tertiary care hospital," Indian Journal of 
Sexually Transmitted Diseases and AIDS, vol. 31, no. 2, pp. 104-108, 2010.

[21] M. L. Grijsen, S. M. Graham, M. Mwangome et al., "Screening for genital and anorectal sexually transmitted infections in HIV prevention trials in Africa," Sexually Transmitted Infections, vol. 84, no. 5, pp. 364-370, 2008.

[22] T. E. Wi, F. J. Ndowa, C. Ferreyra et al., "Diagnosing sexually transmitted infections in resource-constrained settings: challenges and ways forward," Journal of the International AIDS Society, vol. 22, no. S6, Article ID e25343, 2019. 